\title{
General features of spontaneous baryogenesis
}

\author{
Elena Arbuzova ${ }^{\star}$ \\ ${ }^{1}$ Department of Higher Mathematics, Dubna State University, 141980 Dubna, Russia, \\ ${ }^{2}$ Novosibirsk State University, 630090 Novosibirsk, Russia
}

\begin{abstract}
The classical version of spontaneous baryogenesis is studied in details. It is shown that the relation between the time derivative of the (pseudo)goldstone field and the baryonic chemical potential essentially depends upon the representation chosen for the fermionic fields with non-zero baryonic number (quarks). The kinetic equation, used for the calculations of the cosmological baryon asymmetry, is generalized to the case of non-stationary background. The effects of the finite interval of the integration over time are also included into consideration.
\end{abstract}

\section{Introduction}

The Standard Model of cosmology (also called Standard Cosmological Model) is currently the best theory for the description of the Universe. It is based on two fundamental ingredients: the Standard Model of particle physics, which is used for the matter content, and General Relativity, which describes gravitational interactions. It also requires the inflationary paradigm, which is an elegant mechanism to fix a few problems of the scenario. The Standard Model of cosmology very successfully explains a huge amount of observational data, including as the most remarkable ones Hubble's law, the primordial abundances of light elements, and the cosmic microwave background.

However, there are several puzzles that strongly suggest to look for new physics. It is striking, that only $5 \%$ of the Universe is made of the known matter (mainly protons and neutrons). About $25 \%$ of the Universe is likely made of some weakly interactive particles not belonging to the Minimal Standard Model of particle physics. For the time being this component is called dark matter. The other $70 \%$ of the Universe is really a mystery: it looks like a uniformly distributed perfect fluid with an unusual equation of state $P=-\rho$, where $P$ is the pressure and $\rho$ is the energy density, and it is responsible for the present accelerated expansion rate of the Universe. It is usually indicated with the name dark energy, but its origin is not clear at all and a breakdown of General Relativity at large scales is also a possibility. The inflation mechanism cannot be cast in the framework of the Standard Model of particle physics and there are also some possible alternative scenarios that fix the Standard Model of cosmology in a different way. Lastly, we do not understand the origin of the matter-antimatter asymmetry around us. The local Universe is clearly matter dominated, but such an asymmetry cannot be created within the Minimal Standard Model of particle physics.

The term "baryogenesis" is used to indicate the generation of the asymmetry between baryons and antibaryons. In 1967, Andrey Sakharov pointed out three ingredients, today known as Sakharov

^e-mail: arbuzova@uni-dubna.ru 
principles [1], to produce a matter-antimatter asymmetry from an initially symmetric Universe. These conditions include: a) non-conservation of baryonic number; b) breaking of symmetry between particles and antiparticles; c) deviation from thermal equilibrium. (For the details see e.g. the reviews [2, 3]). However, none of these conditions is obligatory. Of particular interest is the scenario of spontaneous baryogenesis (SBG) [4], which can proceed in thermal equilibrium, moreover, it is usually most efficient in thermal equilibrium. The term "spontaneous" is related to spontaneous breaking of underlying symmetry of the theory.

It is assumed that in the unbroken phase the theory is invariant with respect to the global $U(1)$ symmetry, which ensures conservation of baryonic number. This symmetry is spontaneously broken and in the broken phase the Lagrangian density acquires the term

$$
\mathcal{L}_{S B}=\left(\partial_{\mu} \theta\right) J_{B}^{\mu},
$$

where $\theta$ is the Goldstone field and $J_{B}^{\mu}$ is the baryonic current. Due to the spontaneous symmetry breaking (SSB) this current is not conserved. The next step is the statement that the Hamiltonian density corresponding to $\mathcal{L}_{S B}$ is simply the Lagrangian density taken with the opposite sign:

$$
\mathcal{H}_{S B}=-\mathcal{L}_{S B}=-\left(\partial_{\mu} \theta\right) J_{B}^{\mu} .
$$

For the spatially homogeneous field $\theta=\theta(t)$ this Hamiltonian is reduced to $\mathcal{H}_{S B}=-\dot{\theta} n_{B}$, where $n_{B} \equiv J_{B}^{4}$ is the baryonic number density, so it is tempting to identify $\dot{\theta}$ with the chemical potential, $\mu$, of the corresponding system. If this is the case, then in thermal equilibrium the baryon asymmetry would evolve to:

$$
n_{B}=\frac{g_{S} B_{Q}}{6}\left(\mu T^{2}+\frac{\mu^{3}}{\pi^{2}}\right) \rightarrow \frac{g_{S} B_{Q}}{6}\left(\dot{\theta} T^{2}+\frac{\dot{\theta}^{3}}{\pi^{2}}\right),
$$

where $T$ is the cosmological plasma temperature, $g_{S}$ and $B_{Q}$ are respectively the number of the spin states and the baryonic number of quarks, which are supposed to be the bearers of the baryonic number.

In presented work the classical version of spontaneous baryogenesis is studied [5]. The talk is organized as follows. In Section 2 the general features of the spontaneous breaking of baryonic $U(1)$ symmetry are described, the equations of motion for the quarks fields and for the (pseudo)Goldstone mode are presented. In Sec. 3 the baryonic chemical potential is introduced, and the baryon number density is calculated in equilibrium with respect to baryo-nonconserving interactions for different representations of the quark fields. Next, in Sec. 4 the standard kinetic equation in stationary background is presented. In Sec. 5 we derive kinetic equation in time dependent external field and/or for the case when energy is not conserved because of finite limits of integration over time. Several examples, when such kinetic equation is relevant, are presented in Sec. 6. Lastly in Sec. 7 we conclude.

\section{Spontaneous symmetry breaking and goldstone mode}

Let us consider the theory of complex scalar field $\Phi$ interacting with "quarks", $Q$, and "leptons", $L$, with the Lagrangian:

$$
\mathcal{L}(\Phi)=g^{\mu v} \partial_{\mu} \Phi^{*} \partial_{\nu} \Phi-V\left(\Phi^{*} \Phi\right)+\bar{Q}\left(i \gamma^{\mu} \partial_{\mu}-m_{Q}\right) Q+\bar{L}\left(i \gamma^{\mu} \partial_{\mu}-m_{L}\right) L+\mathcal{L}_{i n t}(\Phi, Q, L),
$$

where $\mathcal{L}_{\text {int }}$ describes the interaction between $\Phi$ and fermionic fields. In the toy model studied below we take it in the form:

$$
\mathcal{L}_{\text {int }}=\frac{\sqrt{2}}{m_{X}^{2}} \frac{\Phi}{f}\left(\bar{L} \gamma_{\mu} Q\right)\left(\bar{Q}^{c} \gamma_{\mu} Q\right)+\text { h.c. },
$$


where $Q^{c}$ is charged conjugated quark spinor, $m_{X}$ is a parameter with dimension of mass, and $f$ is related to the vacuum expectation value of $\Phi$ defined below in Eq. (6). Such an interaction can appear e.g. in $S U(5)$ Grand Unified Theory. For simplicity, in our toy model we do not take into account the quark colors.

$Q$ and $L$ can be any fermions, not necessarily quarks and leptons of the Standard Model. They can be e.g. new heavy fermions possessing similar or the same quantum numbers as the quarks and leptons of the Standard Model and may couple to the ordinary quarks and leptons.

The global $U(1)$-symmetry is assumed to be spontaneously broken at the energy scale $f$ via the potential of the form:

$$
V\left(\Phi^{*} \Phi\right)=\lambda\left(\Phi^{*} \Phi-f^{2} / 2\right)^{2}
$$

This potential reaches minimum at the vacuum expectation value of $\Phi$ equal to $\langle\Phi\rangle=f e^{i \phi_{0} / f} / \sqrt{2}$ with an arbitrary constant phase $\phi_{0}$.

Below scale $f$ we can neglect the heavy radial mode of $\Phi$ with the mass $m_{\text {radial }}=\lambda^{1 / 2} f$, since being very massive it is frozen out, but this simplification is not necessary and is not essential for the baryogenesis. The remaining light degree of freedom is the variable field $\phi$, which is the Goldstone boson of the spontaneously broken $U(1)$. Up to a constant factor the field $\phi$ is the angle around the bottom of the Mexican hat potential given by eq. (6). Correspondingly we introduce the dimensionless angular field $\theta \equiv \phi / f$ and thus $\Phi=\langle\Phi\rangle \exp (i \theta)$.

The low energy limit of the Lagrangian (4) in the broken phase, which effectively describes the dynamics of $\theta$-field, has the form:

$$
\begin{aligned}
\mathcal{L}_{1}(\theta)=\frac{f^{2}}{2} \partial_{\mu} \theta \partial^{\mu} \theta+ & \bar{Q}_{1}\left(i \gamma^{\mu} \partial_{\mu}-m_{Q}\right) Q_{1}+\bar{L}\left(i \gamma^{\mu} \partial_{\mu}-m_{L}\right) L+ \\
& \left(\frac{e^{i \theta}}{m_{X}^{2}}\left(\bar{L} \gamma_{\mu} Q_{1}\right)\left(\bar{Q}_{1}^{c} \gamma_{\mu} Q_{1}\right)+\text { h.c. }\right)-U(\theta) .
\end{aligned}
$$

Here we added the potential $U(\theta)$, which may be induced by an explicit symmetry breaking and can lead, in particular, to a nonzero mass of $\theta$. We use the notation $Q_{1}$ for the quark field to distinguish it from the phase rotated field $Q_{2}$ introduced below in Eq. (9). In a realistic model the quark fields should be (anti)symmetrized with respect to color indices, omitted here for simplicity.

If $U(\theta)=0$, the theory still remains invariant under the global transformations (i.e. with $\alpha=$ const):

$$
Q \rightarrow e^{-i \alpha / 3} Q, \quad L \rightarrow L, \quad \theta \rightarrow \theta+\alpha .
$$

If we only rotate the quark field as above but with coordinate dependent $\alpha=\theta(t, \mathbf{x})$, introducing the new field $Q_{1}=e^{-i \theta / 3} Q_{2}$, then the Lagrangian (7) is transformed into:

$$
\begin{array}{r}
\mathcal{L}_{2}(\theta)=\frac{f^{2}}{2} \partial_{\mu} \theta \partial^{\mu} \theta+\bar{Q}_{2}\left(i \gamma^{\mu} \partial_{\mu}-m_{Q}\right) Q_{2}+\bar{L}\left(i \gamma^{\mu} \partial_{\mu}-m_{L}\right) L+ \\
\left(\frac{1}{m_{X}^{2}}\left(\bar{Q}_{2} \gamma_{\mu} L\right)\left(\bar{Q}_{2} \gamma_{\mu} Q_{2}^{c}\right)+\text { h.c. }\right)+\left(\partial_{\mu} \theta\right) J^{\mu}-U(\theta),
\end{array}
$$

where the quark baryonic current is $J_{\mu}=(1 / 3) \bar{Q} \gamma_{\mu} Q$. Note that the current has the same form in terms of $Q_{1}$ and $Q_{2}$.

The equation of motion for the quark field $Q_{1}$ obtained from Lagrangian (7) has the form:

$$
\left(i \gamma^{\mu} \partial_{\mu}-m_{Q}\right) Q_{1}+\frac{e^{-i \theta}}{m_{X}^{2}}\left[\gamma_{\mu} L\left(\bar{Q}_{1} \gamma_{\mu} Q_{1}^{c}\right)+2 \gamma_{\mu} Q_{1}^{c}\left(\bar{Q}_{1} \gamma_{\mu} L\right)\right]=0 \text {. }
$$


Analogously the equation of motion for the phase rotated field $Q_{2}$ derived from Lagrangian (9) is:

$$
\left(i \gamma^{\mu} \partial_{\mu}-m_{Q}+\frac{1}{3} \gamma^{\mu} \partial_{\mu} \theta\right) Q_{2}+\frac{1}{m_{X}^{2}}\left[\gamma_{\mu} L\left(\bar{Q}_{2} \gamma_{\mu} Q_{2}^{c}\right)+2 \gamma_{\mu} Q_{2}^{c}\left(\bar{Q}_{2} \gamma_{\mu} L\right)\right]=0 \text {. }
$$

Equations for $\theta$-field derived from these two Lagrangians in flat space-time have respectively the forms:

$$
f^{2}\left(\partial_{t}^{2}-\Delta\right) \theta+U^{\prime}(\theta)+\left[\frac{i e^{-i \theta}}{m_{X}^{2}}\left(\bar{Q}_{1} \gamma_{\mu} L\right)\left(\bar{Q}_{1} \gamma_{\mu} Q_{1}^{c}\right)+\text { h.c. }\right]=0
$$

and

$$
f^{2}\left(\partial_{t}^{2}-\Delta\right) \theta+U^{\prime}(\theta)+\partial_{\mu} J_{B}^{\mu}=0
$$

where $U^{\prime}(\theta)=d U / d \theta$.

Using either the equation of motion (10) or (11) we can check that the baryonic current is not conserved. Indeed, its divergence is:

$$
\partial_{\mu} J_{B}^{\mu}=\frac{i e^{-i \theta}}{m_{X}^{2}}\left(\bar{Q}_{1} \gamma_{\mu} Q_{1}^{c}\right)\left(\bar{Q}_{1} \gamma^{\mu} L\right)+\text { h.c. }
$$

(and similarly for $Q_{2}$ but without the factor $\exp (-i \theta)$ ). So the equations of motion for $\theta$ in both cases (12) and (13) coincide, as expected.

Eq. (13) expresses the law of the total baryonic current conservation in the unbroken phase. When the symmetry is broken, the non-conservation of the physical baryons (in our case of "quarks") becomes essential and may lead to the observed cosmological baryon asymmetry. Such B-non conserving interaction may have many different forms. The one presented above describes transition of three quark-type fermions into (anti)lepton. There may be transformation of two or three quarks into equal number of antiquarks. Such interaction describes neutron-antineutron oscillations, now actively looked for [6]. There even can be a "quark" transition into three "leptons". Depending on the interaction type the relation between $\dot{\theta}$ and the effective chemical potential would have different forms (see below).

In the spatially homogeneous case, when $\partial_{\mu} J_{B}^{\mu}=\dot{n}_{B}$ and $\theta=\theta(t)$, and if $U(\theta)=0$, equation (13) can be easily integrated giving:

$$
f^{2}\left[\dot{\theta}(t)-\dot{\theta}\left(t_{i n}\right)\right]=-n_{B}(t)+n_{B}\left(t_{i n}\right) .
$$

It is usually assumed that the initial baryon asymmetry vanishes, $n_{B}\left(t_{i n}\right)=0$.

The evolution of $n_{B}(t)$ is governed by the kinetic equation discussed in Sec. 4, which allows to express $n_{B}$ through $\theta(t)$ and thus to obtain the closed systems of, generally speaking, integrodifferential equations. In thermal equilibrium the relation between $\dot{\theta}$ and $n_{B}$ may become an algebraic one, but this is true only in the case when the integration over time is sufficiently long and if $\dot{\theta}$ is constant or slowly varying function of time.

In the cosmological Friedmann-Robertson-Walker (FRW) background the equation of motion of $\theta$ (13) becomes:

$$
f^{2}\left(\partial_{t}+3 H\right) \dot{\theta}-a^{-2}(t) \Delta \theta+U^{\prime}(\theta)=-\left(\partial_{t}+3 H\right) n_{B},
$$

where $a(t)$ is the cosmological scale factor and $H=\dot{a} / a$ is the Hubble parameter. For the homogeneous theta-field, $\theta=\theta(t)$, this equation turns into:

$$
f^{2}\left(\partial_{t}+3 H\right) \dot{\theta}+U^{\prime}(\theta)=-\left(\partial_{t}+3 H\right) n_{B}
$$

We do not include the curvature effects into the Dirac equations because this is not necessary for what follows. Still we are using expression for the current divergence in the form $\mathcal{D}_{\mu} J^{\mu}=\dot{n}_{B}+3 H n_{B}$, but not just $\dot{n}_{B}$. 


\section{Kinetic equilibrium}

The study of kinetics of fermions in the cosmological background is grossly simplified if the particles are in equilibrium with respect to elastic scattering, to their possible annihilation e.g. into photons, and to other baryo-conserving interactions. The equilibrium with respect to elastic scattering implies the following form of the phase space distribution functions:

$$
f_{e q}=[1+\exp (E / T-\xi)]^{-1},
$$

where the dimensionless chemical potential $\xi=\mu / T$ has equal magnitude but opposite signs for particles and antiparticles. The baryonic number density for small $\xi$ is usually given by the expression

$$
n_{B}=g_{S} B_{Q} \xi_{B} T^{3} / 6
$$

(compare to eq. (3)). Here $\xi_{B}$ is the baryonic chemical potential. This equation which expresses baryonic number density through chemical potential is true only for the normal relation between the energy and three-momentum, $E=\sqrt{p^{2}+m^{2}}$, with equal masses of particles and antiparticles.

Vanishing baryon asymmetry implies $\xi_{B}=0$, as is usually the case. If the baryonic number of quarks is conserved, $n_{B}$ remains constant in the comoving volume and it means in turn that $\xi=$ const for massless particles. If $n_{B}=0$ initially, then $\xi_{B}$ remains identically zero. If baryonic number is not conserved, then as we see below from the kinetic equation, equilibrium with respect to Bnonconserving processes leads to $\xi_{B}=c \dot{\theta} / T$, as is envisaged by SBG. The constant $c$ depends upon the concrete type of reaction. Complete thermal equilibrium in the standard theory demands $n_{B} \rightarrow 0$, but a deviation from thermal equilibrium of B-nonconserving interaction leads to generation of nonzero $\xi_{B}$ and correspondingly to non-zero $n_{B}$.

The situation changes, if quarks and antiquarks satisfy the equation of motion (11), for which the following dispersion relation is valid

$$
E=\sqrt{p^{2}+m^{2}} \mp \dot{\theta} / 3
$$

where the signs $\mp$ refer to particles or antiparticles respectively. So the energies of quarks and antiquarks with the same three-momentum are different. This is similar to mass difference which may be induced by CPT violation. It is noteworthy that the above dispersion relation is derived under assumption of constant or slow varying $\dot{\theta}$. Otherwise the Fourier transformed Dirac equation cannot be reduced to the algebraic one and the particle energy is not well defined.

The baryon number density corresponding to the dispersion relation (20) is given by the expression

$$
n_{B} \equiv g_{S} B_{Q} \int \frac{d^{3} p}{(2 \pi)^{3}}[f(p)-\bar{f}(p)]=\frac{g_{S} B_{Q}}{6}\left(\xi_{B}+\frac{\dot{\theta}}{3 T}\right) T^{3} .
$$

where $\bar{f}$ is the distribution function of antiparticles. If the baryon number is conserved and is zero initially, the condition $\xi_{B}+\dot{\theta} /(3 T)=0$ would be always fulfilled. If $\mathrm{B}$ is not conserved, then the equilibrium with respect to B-nonconserving processes demands $\xi_{B}=0$, as it follows from kinetic equation presented below. So evidently $\xi_{B} \neq \dot{\theta}$ but nevertheless the baryon asymmetry is proportional to $\dot{\theta}$ as follows from eq. (21).

\section{Kinetic equation in (quasi)stationary background}

The temporal evolution of the distribution function of i-th type particle, $f_{i}(t, p)$, in an arbitrary process $i+Y \leftrightarrow Z$ in the FRW background, is governed by the equation:

$$
\frac{d f_{i}}{d t}=\left(\partial_{t}-H p_{i} \partial_{p_{i}}\right) f_{i}=I_{i}^{c o l l},
$$


with the collision integral equal to:

$$
\begin{aligned}
& I_{i}^{\text {coll }}=\frac{(2 \pi)^{4}}{2 E_{i}} \sum_{Z, Y} \int d v_{Z} d v_{Y} \delta^{4}\left(p_{i}+p_{Y}-p_{Z}\right) \\
& {\left[|A(Z \rightarrow i+Y)|^{2} \prod_{Z} f \prod_{i+Y}(1 \pm f)-|A(i+Y \rightarrow Z)|^{2} f_{i} \prod_{Y} f \prod_{Z}(1 \pm f)\right],}
\end{aligned}
$$

where $A(a \rightarrow b)$ is the amplitude of the transition from state $a$ to state $b, Y$ and $Z$ are arbitrary, generally multi-particle states, $\left(\prod_{Y} f\right)$ is the product of the phase space densities of particles forming the state $Y$, and

$$
d v_{Y}=\prod_{Y} \overline{d p} \equiv \prod_{Y} \frac{d^{3} p}{(2 \pi)^{3} 2 E} .
$$

The signs ' + ' or '-' in $\prod(1 \pm f)$ are chosen for bosons and fermions respectively. We neglect the effects of space-time curvature in the collision integral, which is generally a good approximation.

We are interested in the evolution of the baryon number density, which is the time component of the baryonic current $J^{\mu}: n_{B} \equiv J^{4}$. Due to the quark-lepton transitions the current is non-conserved and its divergence is given by eq. (14). The similar expression is evidently true in terms of $Q_{2}$ but without the factor $\exp (-i \theta)$. Let us first consider the latter case, when the interaction described by the Lagrangian (9), which contains the product of three "quark" and one "lepton" operators, and take as an example the process $q_{1}+q_{2} \leftrightarrow \bar{q}+l$.

Since the interaction in this representation does not depend on time, the energy is conserved and the collision integral has the usual form with conserved four-momentum. Quarks are supposed to be in kinetic equilibrium but probably not in equilibrium with respect to B-nonconserving interactions, so their distribution functions have the form:

$$
f_{q}=\exp \left(-\frac{E}{T}+\xi_{B}\right) \text { and } f_{\bar{q}}=\exp \left(-\frac{E}{T}-\xi_{B}\right)
$$

Here and in what follows the Boltzmann statistics is used. According to ref. [7], Fermi corrections are typically at the $10 \%$ level. Since the dispersion relation for quarks and antiquarks (20) depends upon $\dot{\theta}$, the baryon asymmetry in this case is given by eq. (21) and the kinetic equation takes the form:

$$
\frac{g_{S} B_{Q}}{6} \frac{d}{d t}\left(\xi_{B}+\frac{\dot{\theta}}{3 T}\right)=-c_{1} \Gamma \xi_{B},
$$

where $c_{1}$ is a numerical factor of order unity and $\Gamma$ is the rate of baryo-nonconserving reactions. If the amplitude of this reaction has the form determined by the Lagrangian (9), then $\Gamma \sim T^{5} / m_{X}^{4}$.

For constant or slow varying temperature the equilibrium solution to this equation is $\xi_{B}=0$ and the baryon number density (21) is proportional to $\dot{\theta}, n_{B}=\left(g_{S} B_{Q} / 18\right) \dot{\theta} T^{2}$, with $\dot{\theta}$ evolving as:

$$
\dot{\theta}=\frac{f^{2}}{f^{2}+g_{S} B_{Q} T^{2} / 18}\left(\frac{T}{T_{i n}}\right)^{3} \dot{\theta}\left(t_{i n}\right) .
$$

We see that the equilibrium value of $n_{B}$ drops down with decreasing temperature as $T^{5}$. However at small temperatures baryon non-conserving processes switch-off and $n_{B}$ tends to a constant value in comoving volume.

Let us check now what happens if the dependence on $\theta$ is moved from the quark dispersion relation to the B-nonconserving interaction term (12). The collision integral (23) contains delta-functions 
imposing conservation of energy and momentum if there is no external field which depends upon coordinates. In our case, when quarks "live" in the $\theta(t)$-field, the collision integral should be modified. Since the interaction proceeds in time dependent background, the energy conservation delta-function does not emerge, and we have now an additional factor, namely, $\exp [ \pm i \theta(t)]$, under the integral by calculation of the amplitude of transition from the initial state $|i n\rangle$ to a final state $\mid$ fin $\rangle$. In general case this integral cannot be taken analytically, but if we can approximate $\theta(t)$ as $\theta(t) \approx \dot{\theta} t$ with a constant or slowly varying $\dot{\theta}$, the integral is simply taken giving e.g. for the process of two quark transformation into antiquark and lepton, $q_{1}+q_{2} \leftrightarrow \bar{q}+l$, the energy balance condition imposed by $\delta\left(E_{q_{1}}+E_{q_{2}}-E_{\bar{q}}-E_{l}-\dot{\theta}\right)$. In other words the energy is non-conserved due to the action of the external field $\theta(t)$. The approximation of linear evolution of $\theta$ with time can be valid if the reactions are fast in comparison with the rate of the $\theta$-evolution.

Returning to our case we can see that the collision integral taken over the three-momentum of the particle under scrutiny (i.e. particle $i$ in eq. (23) ) e.g. for process the $q_{1}+q_{2} \rightarrow l+\bar{q}$ turns into:

$$
\begin{aligned}
& \dot{n}_{B}+3 H n_{B} \sim \\
& \int d \tau_{l \bar{q}} d \tau_{q_{1} q_{2}}|A|^{2} \delta\left(E_{q_{1}}+E_{q_{2}}-E_{l}-E_{\bar{q}}-\dot{\theta}\right) \delta\left(\mathbf{P}_{i n}-\mathbf{P}_{f i n}\right) e^{-E_{i n} / T}\left(e^{\xi_{L}-\xi_{B}+\dot{\theta} / T}-e^{2 \xi_{B}}\right),
\end{aligned}
$$

where $d \tau_{l, \bar{q}}=d^{3} p_{l} d^{3} p_{\bar{q}} /\left[4 E_{l} E_{\bar{q}}(2 \pi)^{6}\right]$. We assumed here that all participating particles are in kinetic equilibrium, i.e. their distribution functions have the form (25). In expression (28) $\xi_{B}$ and $\xi_{L}$ denote baryonic and leptonic chemical potentials respectively and the effects of quantum statistics are neglected but only for brevity of notations. The assumption of kinetic equilibrium is well justified because it is enforced by the very efficient elastic scattering. Another implicit assumption is the usual equilibrium relation between chemical potentials of particles and antiparticles, $\bar{\mu}=-\mu$, imposed e.g. by the fast annihilation of quark-antiquark or lepton-antilepton pairs into two and three photons. Anyhow the assumption of kinetic equilibrium is one of the cornerstones of the spontaneous baryogenesis.

The conservation of $(B+L)$ implies the following relation: $\xi_{L}=-\xi_{B} / 3$. Keeping this in mind, we find

$$
\dot{n}_{B}+3 H n_{B} \approx-\left(1-e^{\dot{\theta} / T-3 \xi_{B}+\xi_{L}}\right) I \approx\left(\frac{\dot{\theta}}{T}-\frac{10}{3} \xi_{B}\right) I,
$$

where we assumed that $\xi_{B}$ and $\dot{\theta} / T$ are small. In relativistic plasma with temperature $T$ the factor $I$, coming from the collision integral, can be estimated as $I=T^{8} / \mathrm{m}^{4}$, where $m$ is a numerical constant with dimension of mass. It differs from $m_{X}$, introduced in eq. (7), by a numerical coefficient.

For a large factor $I$ we expect the equilibrium solution

$$
\xi_{B}=\frac{3}{10} \frac{\dot{\theta}}{T},
$$

so $\dot{\theta}$ up to the numerical factor seems to be the baryonic chemical potential, as expected in the usually assumed SBG scenario. The value of the coefficient $c=3 / 10$ in Eq. (30) may be different for other types of B-nonconcerving reactions, e.g. for the reaction $3 q \leftrightarrow 3 \bar{q}$ one can find that $c=1 / 6$. Let us remind that for the dispersion relation (20) the baryonic chemical potential is not proportional to $\dot{\theta}(t)$, but is equal to zero, see Eq. (26) and comments below.

\section{Kinetic equation for time-varying amplitude}

The canonical kinetic equation (22) is usually presented for scattering or decay processes in time independent or slowly varying background with the collision integral giving by eq. (23). 
As it was mentioned above, if the interaction proceeds in time dependent background and/or the time duration of the process is finite, then the energy conservation delta-function does not emerge, so one has to make the time integration with an account of time-varying background and integrate over the phase space without energy conservation.

In what follows we consider two-body inelastic process with baryonic number non-conservation with the amplitude obtained from the last term in Lagrangian (7). At the moment we will not specify the concrete form of the reaction but only will say that it is the two-body reaction

$$
a+b \leftrightarrow c+d,
$$

where $a, b, c$, and $d$ are some quarks and leptons or their antiparticles. The expression for the evolution of the baryonic number density, $n_{B}$, follows from eq. (22) after integration of its both sides over $d^{3} p_{i} /(2 \pi)^{3}$. Thus we obtain:

$$
\dot{n}_{B}+3 H n_{B}=-\frac{(2 \pi)^{3}}{t_{\max }} \int d v_{i n} d v_{f i n} \delta\left(\mathbf{P}_{i n}-\mathbf{P}_{f i n}\right)|A|^{2}\left(f_{a} f_{b}-f_{c} f_{d}\right)
$$

where e.g. $d v_{i n}=d^{3} p_{a} d^{3} p_{b} /\left[4 E_{a} E_{b}(2 \pi)^{6}\right]$ and the amplitude of the process is defined as

$$
A=\left(\int_{0}^{t_{\max }} d t e^{i\left[\left(E_{c}+E_{d}-E_{a}-E_{b}\right) t+\theta(t)\right]}\right) F\left(p_{a}, p_{b}, p_{c}, p_{d}\right),
$$

and $F$ is a function of 4-momenta of the participating particles, determined by the concrete form of the interaction Lagrangian. In what follows we consider two possibilities: $F=$ const and $F=\psi^{4} m_{X}^{-2}$, where in the last case $\psi^{4}$ symbolically denotes the product of the Dirac spinors of particles $a, b, c, d$.

In the case of equilibrium with respect to baryon conserving reactions the distribution functions have the canonical form $f_{a}=\exp \left(-E_{a} / T+\xi_{a}\right)$, where $\xi_{a} \equiv \mu_{a} / T$ is the dimensionless chemical potential. So for constant $F$ the product $|A|^{2}\left(f_{a} f_{b}-f_{c} f_{d}\right)$ depends upon the particle 4-momenta only through $E_{\text {in }}$ and $E_{\text {fin }}$, where

$$
E_{\text {in }}=E_{a}+E_{b} \text {, and } E_{\text {fin }}=E_{c}+E_{d} .
$$

Note that the amplitude A (33) depends only on $E_{-}=E_{\text {in }}-E_{\text {fin }}$ but not on $E_{+}=E_{\text {in }}+E_{\text {fin }}$, while the products of the particle densities in the phase space are

$$
f_{a} f_{b}=\exp \left(-\frac{E_{+}+E_{-}}{2 T}+\xi_{a}+\xi_{b}\right) \text { and } f_{c} f_{d}=\exp \left(-\frac{E_{+}-E_{-}}{2 T}+\xi_{c}+\xi_{d}\right) .
$$

Now we can perform almost all (but one) integrations over the phase space in eq. (32). Collecting all the factors (35), we finally obtain:

$$
\dot{n}_{B}+3 H n_{B}=-\frac{T^{5}}{2^{5} \pi^{6} t_{\max }} \int_{0}^{\infty} d y\left[e^{\xi_{a}+\xi_{b}}\left(\left|A_{+}\right|^{2}+\left|A_{-}\right|^{2} e^{-y}\right)-e^{\xi_{c}+\xi_{d}}\left(\left|A_{-}\right|^{2}+\left|A_{+}\right|^{2} e^{-y}\right)\right],
$$

where $A_{+}$is the amplitude taken at positive $E_{-}$, while $A_{-}$is taken at negative $E_{-}$. With the substitution $E_{-} \rightarrow\left|E_{-}\right|$the only difference between $A_{+}$and $A_{-}$is that $A_{-}(\theta)=A_{+}(-\theta)$.

The equilibrium is achieved when the integral in eq. (36) vanishes. This point determines the equilibrium values of the chemical potentials in external $\dot{\theta}$ field. Clearly it takes place at:

$$
\xi_{a}+\xi_{b}-\xi_{c}-\xi_{d}=\frac{\left\langle\left|A_{+}\right|^{2} e^{-y}+\left|A_{-}\right|^{2}\right\rangle}{\left\langle\left|A_{+}\right|^{2}+\left|A_{-}\right|^{2} e^{-y}\right\rangle}-1,
$$

where the angular brackets mean integration over $d y$ as indicated in eq. (36). 


\section{Examples of time-varying $\theta$}

\subsection{Constant $\dot{\theta}$}

This is the case usually considered in the literature and the simplest one. The integral (33) is taken analytically resulting in:

$$
|A|^{2} \sim \frac{2-2 \cos \left[\left(\dot{\theta}-E_{-}\right) t_{\max }\right]}{\left(\dot{\theta}-E_{-}\right)^{2}}
$$

where $E_{-}$is running over the positive semi-axis.

For large $t_{\max }$ this expression tends to $\delta\left(E_{-}-\dot{\theta}\right)$, so $\left|A_{+}\right|^{2}=2 \pi \delta\left(E_{-}-\dot{\theta}\right) t_{\max }$ and $\left|A_{-}\right|^{2}=2 \pi \delta\left(E_{-}+\right.$ $\dot{\theta}) t_{\max }=0$, if $\dot{\theta}>0$ and vice versa otherwise. Hence the equilibrium solution is

$$
\xi_{a}+\xi_{b}-\xi_{c}-\xi_{d}-\dot{\theta} / T=0,
$$

coinciding with the standard result.

The limit of $\dot{\theta}=$ const corresponds to the energy non-conservation by the rise (or drop) of the energy of the final state in reaction (31) exactly by $\dot{\theta}$. However if $t_{\max }$ is not sufficiently large, the nonconservation of energy is not equal to $\dot{\theta}$ but somewhat spread out and the equilibrium solution would be different. There is no simple analytical expression in this case, so we have to take the integrals over $y$ in eq. (37) numerically.

The results of the calculations are presented in Fig 1. In the left panel the values of the r.h.s. of eq. (37) are presented as a function of $\dot{\theta} / T$ for the cut-off of the time integration in eq. (38) equal to: $\tau \equiv t_{\max } T=30 ; 10 ; 3$. The larger is the integration time the closer are the lines to $\dot{\theta} / T$, which is also depicted.

In the right panel the relative differences between the r.h.s. of eq. (37) and $\dot{\theta} / T$, normalized to $\dot{\theta} / T$, as a function of $\dot{\theta} / T$ for different maximum time of the integration are presented. We see that for $\tau=30$ the deviations are less than $10 \%$, while for $\tau=3$ the deviations are about $30 \%$. If we take $\tau$ close to unity, the deviations are about $100 \%$. The value of $\dot{\theta} / T$ is bounded from above by approximately 0.3 because at large $\dot{\theta} / T$ the linear expansion, used in our estimates, is invalid.
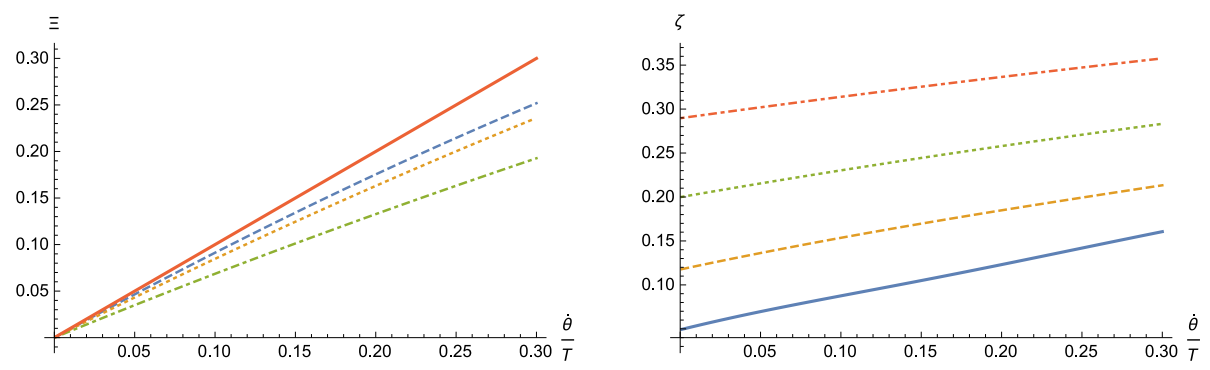

Figure 1. Left: The r.h.s. of eq. (37), denoted by $\Xi$, as a function of $\dot{\theta} / T$ for the cut-off of the time integration in eq. (38): $\tau \equiv t_{\max } T=30$ (dashed); 10 (dotted); 3 (dotdashed) and $\dot{\theta} / T$ (thick). Right: The relative difference: $\zeta=\Xi /(\dot{\theta} / T)-1$, as a function of $\dot{\theta} / T$ for $\tau=30$ (thick), 10 (dashed), 5 (dotted), 3 (dotdashed).

The realistic values of $\tau$ depend upon the model parameters. There is one evident limit related to the cosmological expansion, which implies $\tau<t_{\text {cosm }} T \sim T / H \sim m_{P l} / T$. Here $m_{P l}$ is the Planck mass, $H$ is the Hubble parameter, and $T_{\text {cosm }} \sim 1 / H$, so the effects of the expansion may be significant only near the Planck temperature. Another upper bound on $\tau$ is presented by the kinetic equations 
which demands the characteristic time variation to be close (at least initially) to the inverse reaction rate $\gamma \sim T^{5} / m_{X}^{4}$. The discussed effects would have an essential impact on the approach to equilibrium for $T \sim m_{X}$ which might be realistic.

\subsection{Second order Taylor expansion of $\theta(t)$}

As we have seen in the previous subsection the approximation $\dot{\theta}=$ const is noticeably violated. Here we assume that $\theta(t)$ can be approximated as

$$
\theta(t)=\dot{\theta} t+\ddot{\theta} t^{2} / 2
$$

where $\dot{\theta}$ and $\ddot{\theta}$ are supposed to be constant or slowly varying. In this case the integral over time (33) can also be taken analytically but the result is rather complicated. We need to take the integral

$$
\int_{0}^{t_{\max }} d t \exp [i \theta(t)]
$$

Its real and imaginary parts are easily expressed though the Fresnel functions. So the amplitude squared is given by the functions tabulated in Mathematica and the position of the equilibrium point can be calculated, as in the previous case, by numerical calculation of one dimensional integral.
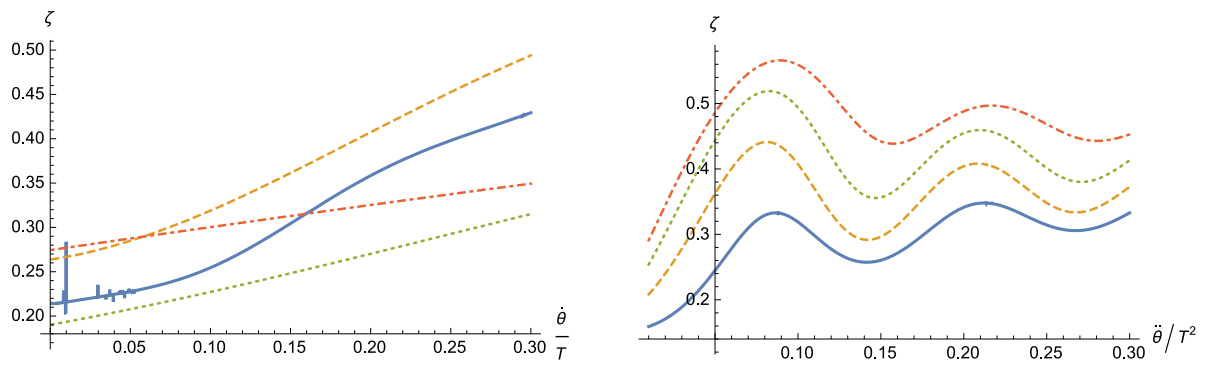

Figure 2. Left: The relative difference: $\zeta=\Xi /(\dot{\theta} / T)-1$, as a function of $\dot{\theta} / T$ for the cut-off of the time integration $\tau=30$ (thick), 10 (dashed), 5 (dotted), 3 (dotdashed) for fixed $\ddot{\theta} / T^{2}=0.1$. Right: The same difference as a function of $\ddot{\theta} / T^{2}$ for fixed time of integration $\tau=10$ and different $\dot{\theta} / T=0.1$ (thick), 0.2 (dashed), 0.3 (dotted), 0.4 (dotdashed).

The r.h.s. of eq. (37) as a function of $\dot{\theta} / T$ for different values of $\tau$ is presented in Fig. 2, at the left panel. It is interesting that the dependence on $\tau$ is non-monotonic. This may be understood by diminishing of the impact of $\ddot{\theta} t^{2}$ at smaller time interval.

To check the dependence on $\ddot{\theta}$ we calculated again the r.h.s. of eq. (37) but now as a function of $\ddot{\theta} / T^{2}$ presented at the right panel of Fig. 2 for fixed time of integration and different values of $\dot{\theta} / T$. We see that the equilibrium point oscillates as a function $\ddot{\theta}$.

\section{Conclusion}

We argue that in the standard description $\dot{\theta}$ is not formally the chemical potential, though in thermal equilibrium $\dot{\theta}$ may tend to the chemical potential with the numerical coefficient which depends upon the model. However, this result is not always true but depends upon the chosen representation of the "quark" fields. In the theory described by the Lagrangian (7) which appears "immediately" after the 
spontaneous symmetry breaking, $\theta(t)$ directly enters the interaction term and in equilibrium $\mu_{B} \sim \dot{\theta}$ indeed. On the other hand, if we transform the quark field, so that the dependence on $\theta$ is shifted to the bilinear product of the quark fields (9), then chemical potential in equilibrium does not tend to $\dot{\theta}$, but to zero. Still, the magnitude of the baryon asymmetry in equilibrium is always proportional to $\dot{\theta}$.

The assumption of a constant or slowly varying $\dot{\theta}$, which is usually done in the SBG scenario, may be not fulfilled and to include the effects of an arbitrary variation of $\theta(t)$, as well as the effects of the finite time integration, we transformed the kinetic equation in such a way that it becomes operative in non-stationary background. A shift of the equilibrium value of the baryonic chemical potential due to this effect is numerically calculated.

In spite of these corrections to the standard SBG scenario, it remains a viable mechanism for creation of the observed cosmological excess of matter over antimatter. However, this mechanism is not particularly efficient in the case of pure spontaneous symmetry breaking, when the potential of the $\theta$-field is absent. Non-zero potential $U(\theta)$, which can appear as a result of an explicit breaking of the baryonic $U(1)$-symmetry in addition to the spontaneous breaking may grossly enhance the efficiency of the spontaneous baryogenesis. The evaluation of the efficiency demands numerical solution of the ordinary differential equation of motion for the $\theta$-field together with the integral kinetic equation. In the case of thermal equilibrium the kinetic equation is reduced to an algebraic one and the system is trivially investigated. The out-of-equilibrium situation is much more complicated technically and will be studied elsewhere.

We assumed that the symmetry breaking phase transition in the early universe occurred instantly. It may be a reasonable approximation, but still the corrections can be significant. This can be also a subject of future work.

Acknowledgement. This work was supported by the Grant of President of Russian Federation for the leading scientific schools of the Russian Federation, NSh-9022.2016.2.

\section{References}

[1] A.D. Sakharov, Pis'ma ZhETF, 5 (1967) 32.

[2] A.D.Dolgov, Phys. Repts 222 (1992) No. 6; A.D. Dolgov, Surveys in High Energy Physics, 13 (1998) 83, hep-ph/9707419.

[3] V.A. Rubakov, M.E. Shaposhnikov, Usp. Fiz. Nauk, 166 (1996) 493, hep-ph/9603208;

A. Riotto, M. Trodden, Ann. Rev. Nucl. Part. Sci. 49 (1999), 35, hep-ph/9901362;

M. Dine, A. Kusenko, Rev. Mod. Phys. 76 (2004) 1.

[4] A. Cohen, D. Kaplan, Phys. Lett. B 199, 251 (1987);

A. Cohen, D. Kaplan, Nucl.Phys. B 308 (1988) 913;

A. G. Cohen, D.B. Kaplan, A.E. Nelson, Phys.Lett. B 263 (1991) 86;

A. G. Cohen, D.B. Kaplan, A.E. Nelson, Phys.Lett. B 336 (1994) 41, hep-ph/940634.

[5] E.V. Arbuzova, A.D. Dolgov, V.A. Novikov, Phys. Rev. D 94 (2016) 123501, arXiv:1607.01247.

[6] D.G. Phillips, II, et al, Phys.Rept. 612 (2016) 1-45.

[7] A.D. Dolgov, K. Kainulainen, Nucl.Phys. B402 (1993) 349-359. 